Revista

Actualidades Investigativas

en Educación

\title{
Validación de una escala de prejuicios hacia personas con discapacidad
}

\author{
Validation of a scale of prejudices towards \\ disabled people
}

\author{
Volumen 20, Número 2 \\ Mayo - Agosto \\ pp. 1-25
}

\begin{abstract}
Alicia Gurdián Fernández
Marie-Claire Vargas Dengo

Carmen Delgado Álvarez

Andrés Sánchez Prada
\end{abstract}

Citar este documento según modelo APA

Gurdián-Fernandez, Alicia., Vargas Dengo, Marie-Claire., Delgado Álvarez, Carmen. y Sánchez Prada, Andrés. (2020). Validación de una escala de prejuicios hacia personas con discapacidad. Revista Actualidades Investigativas en Educación, 20(2), 1-25. Doi. 10.15517/aie.v20i2.41655 


\title{
Validación de una escala de prejuicios hacia personas con discapacidad \\ Validation of a scale of prejudices towards disabled people
}

\author{
Alicia Gurdián Fernández ${ }^{1}$ \\ Marie-Claire Vargas Dengo 2 \\ Carmen Delgado Álvarez 3 \\ Andrés Sánchez Prada 4
}

\begin{abstract}
Resumen: La identificación de prejuicios en la población universitaria, con respecto a las personas con discapacidad, es una necesidad para poder diseñar políticas inclusivas adecuadas tendentes a erradicar las barreras que impiden una educación universal no discriminativa. En la actualidad, no se dispone de un instrumento de evaluación específico que permita realizar una evaluación de necesidades para la actualización, capacitación o formación en las instituciones universitarias de la población con discapacidad. El objetivo del artículo fue desarrollar una escala psicométrica específica, para el ámbito universitario, que permita cuantificar el prejuicio hacia las personas con discapacidad que garantizara su fiabilidad y evidencias de validez. Desde el enfoque cuantitativo de la construcción de escalas psicométricas, se elaboró una batería de ítems a partir del modelo de prejuicio ambivalente y de un estudio cualitativo previo realizado en los años 2017 y 2018. De acuerdo con las valoraciones de jueces expertos, se diseñó una escala inicial de 72 ítems, que fue administrada a una muestra incidental de 551 personas procedentes de tres instituciones universitarias: Universidad de Costa Rica y Universidad Nacional (Costa Rica), y Universidad Pontificia de Salamanca (España). Tras el análisis psicométrico se obtuvo una escala de 24 ítems con una consistencia interna de .89. El Análisis Factorial Confirmatorio muestra índices de ajuste adecuados, (GFI > .95; AGFI > .90; NFI > .95) para un modelo de 4 factores: Idealización benevolente, Evitación de contacto, Amplificación sexista del prejuicio y Percepción de exceso de demandas. Los resultados permiten concluir que esta escala tiene una fiabilidad satisfactoria, además de suficientes evidencias de validez basadas en el contenido y en la estructura interna.
\end{abstract}

Palabras clave: prejuicio, discapacidad, género, educación superior

Abstract: The identification of prejudices towards people with disabilities that underlie the university population is a necessity in order to be able to design appropriate inclusive policies aimed to eradicate barriers to a nondiscriminatory universal education. At present, however, there is no a specific assessment instrument available for this population, which allows for a needs assessment for updating, training or academic formal training in university institutions. The objective of the project was to develop a specific psychometric scale, for the university setting, that would allow quantifying prejudice towards people with disabilities that would guarantee their reliability and validity evidence. From the quantitative approach of the construction of psychometric scales, a battery of items was developed from a previous qualitative study done in 2017 and 2018 and the ambivalent prejudice model. Based on previous assessments by expert judges, an initial scale of 72 items was designed and administered to an incidental sample of 551 people, from three university institutions: UCR and UNA (Costa Rica) and UPSA (Spain). After the psychometric analysis, a 24-items scale was obtained with an internal consistency of .89. The Confirmatory Factor Analysis shows adequate adjustment rates GFI > .95; AGFI > .90; NFI > .95) for a 4factor model: Benevolent Idealization, Contact Avoidance, Sexist Prejudice Amplification, and Perception of Excess Demands. The results allow us to conclude that this scale has satisfactory reliability, and sufficient evidence of validity based on the content and internal structure.

Key Words: prejudice, disability, gender, higher education

${ }_{1}$ Investigadora Jubilada de la Universidad de Costa Rica, en el Instituto de Investigación en Educación, Costa Rica. Dirección electrónica: agurdianf@gmail.com Orcid https://orcid.org/0000-0001-8752-8200

2Investigadora de la Universidad Nacional (UNA), Costa Rica. Dirección electrónica: marie.vargas.dengo@una.cr ORCID http://orcid.org/0000-0001-8468-9690

3 Investigadora de la Universidad Pontificia de Salamanca, en la Facultad de Psicología, España. Dirección electrónica: mcdelgadoal@upsa.es ORCID https://orcid.org/0000-0002-6757-6317

${ }^{4}$ Investigaddor de la Universidad Pontificia de Salamanca, en la Facultad de Psicología, España.

Dirección electrónica: asanchezpr@upsa.es Orcid https://orcid.org/0000-0002-6242-1236

Artículo recibido: 16 de octubre, $2019 \quad$ Aprobado: 20 de abril, 2020 


\section{Introducción}

El propósito de este artículo es describir el procedimiento de construcción de un instrumento, tipo escala, que pueda ser utilizado para identificar prejuicios hacia las personas con discapacidad $(\mathrm{PcD})$ en la población académica, administrativa y estudiantil en instituciones de educación superior. El interés de contar con un instrumento de esta índole radica, básicamente, en que las instituciones universitarias no cuentan con un instrumento similar, hasta la fecha, según se detectó en una exhaustiva revisión bibliográfica realizada previamente (Vargas, 2013). Esta razón justificó que un equipo de investigadoras e investigadores uniera esfuerzos desde tres (3) instituciones universitarias, dos de ellas en Costa Rica: la Universidad de Costa Rica (UCR) y la Universidad Nacional (UNA), y una en España: la Universidad Pontificia de Salamanca (UPSA). Esto, para diseñar y validar una escala que logre identificar los prejuicios presentes en la población docente, administrativa y estudiantil con respecto a las personas con alguna condición de discapacidad. El proceso investigativo tendiente al diseño y elaboración de la escala se realizó, durante los años 2018 y 2019, por las personas autoras de este artículo.

El objetivo general explícito de la investigación consistió en, primer lugar, obtener un instrumento original, consistente y con evidencias de validez que, eventualmente, se pudiera utilizar para evaluar los prejuicios hacia las personas con discapacidad. En segundo lugar Disponer de este instrumento y de los resultados que, eventualmente, pudiera generar sería útil para proponer políticas institucionales que respondan a las necesidades particulares de la población universitaria que presenta alguna condición de discapacidad. Asímismo, también, podría ser utilizado para planificar actividades académicas de actualización, capacitación o formación para contribuir con la construcción de una cultura universitaria inclusiva.

Partimos de un marco conceptual que concibe las conductas y las actitudes individuales hacia grupos estigmatizados como un resultado de la asimilación del sistema de creencias de la ideología dominante (Quiles del Castillo, 2019). Consecuentemente, las razones que motivan este estudio redundan en la problemática de la existencia y prevalencia de los prejuicios hacia las PcD en las instituciones universitarias (Vargas, 2013). En la actualidad: a) hay quienes afirman que hoy en día se advierte una tendencia a la disminución del prejuicio. Pese a ello, aún hay personas discriminadas por nacionalidad, etnia, sexo, orientación sexual, enfermedades o trastornos mentales, así como por su condición de discapacidad. b) Algunos estudios sobre la discriminación y el prejuicio 
concluyen que este ha disminuido porque los instrumentos tradicionales así lo evidencian. De hecho, las personas suelen afirmar no tener prejuicios y ser mucho más tolerantes o respetuosas de las diferencias; sin embargo, los comportamientos exhibidos no muestran cambios tan significativos como tendemos a creer. c) Es probable que el prejuicio hacia las personas con discapacidad se haya tornado más sutil y silencioso. No obstante, continúa teniendo un impacto negativo sobre las personas con discapacidad (PcD) en el contexto de instituciones educativas. Además, esto también puede ser un indicador de que la cultura de los derechos humanos no ha permeado en las personas prejuiciadas, y que las instituciones universitarias no han sido exitosas en implementar los mandatos de la Convención sobre los derechos de las personas con discapacidad y su Protocolo Facultativo, aprobados el 13 de diciembre de 2006 en la Sede de las Naciones Unidas en Nueva York. Importante señalar que dicha Convención propone un "cambio paradigmático" de las actitudes y enfoques respecto de las personas con discapacidad. ${ }^{5}$

Las interrogantes que genera esta investigación son las siguientes: ¿El prejuicio hacia las PcD es unidimensional? ¿Es plausible proponer un modelo del prejuicio hacia las PcD con base en las dimensiones teóricas abordadas? ¿Cómo se relaciona la forma hostil del prejuicio con la forma benevolente? ¿Tiene un componente de género el constructo prejuicio hacia la discapacidad?

A continuación, se presenta el referente teórico y se abordan los componentes que sustentan esta investigación. Posteriormente, se exponen los aspectos metodológicos, los resultados y las conclusiones a las que se llega.

\section{Andamiaje teórico}

La definición del constructo prejuicio sutil hacia las personas con condición de discapacidad $(\mathrm{PcD})$ se realizó desde las teorías psicosociales sobre actitud (Briñol, Petty, y Stavraki, 2019) y la posibilidad del cambio de actitudes (Albarracín y Shavitt, 2018). Estas teorías relacionan el estereotipo, el prejuicio y la discriminación como los tres componentes

\footnotetext{
5 En ella se adopta una amplia clasificación de las personas con discapacidad y se reafirma que todas las personas con todos los tipos de discapacidad deben poder gozar de todos los derechos humanos y libertades fundamentales. Se aclara y precisa cómo se aplican a las personas con discapacidad todas las categorías de derechos y se indican las esferas en las que es necesario introducir adaptaciones para que las personas con discapacidad puedan ejercer en forma efectiva sus derechos y las esferas en las que se han vulnerado esos derechos $y$ en las que debe reforzarse la protección de los derechos. https://www.un.org/development/desa/disabilities-es/convencion-sobre-los-derechos-de-las-personas-condiscapacidad-2.html
} 
de las actitudes (Fishbein y Ajzen, 1975; Rosenberg y Hovland, 1960), o como elementos interrelacionados entre sí, aunque no formen parte de la actitud como tal (McCartan, et al, 2018). La concepción tripartita define la actitud, como "un conjunto organizado de convicciones o ideas que predispone favorable o desfavorablemente a actuar respecto de un objeto social" (Pallí y Martínez, 2004, p.194). Esto establece tanto la proximidad conceptual como las diferencias entre los tres componentes:

1) Estereotipo o componente cognitivo: conjunto de pensamientos, ideas y creencias sobre el objeto de actitud, que pueden ser favorables o desfavorables. El estereotipo sería este conjunto de creencias que afecta la forma de percibir a las personas clasificadas como parte de un grupo.

2) Prejuicio o componente afectivo: sentimientos o emociones que provoca el objeto de actitud, pueden ser también positivos (agrado) o negativos (desagrado). El prejuicio ya sea de afecto o de evaluación negativa hacia las personas miembros de un grupo, emerge cuando la asignación grupal se realiza en función de la categorización asignada a la persona como miembro de un grupo socialmente estigmatizado.

3) Discriminación o componente comportamental: tendencia o predisposición a actuar acorde con el prejuicio hacia el objeto de actitud. La discriminación es, por tanto, un trato desigual a las personas en función del grupo en el que son categorizadas.

Frente a esta concepción tridimensional de la actitud, el modelo unidimensional (Allport, 1935) considera la actitud como una única dimensión bipolar que determina la posición hacia el objeto de actitud, donde el aspecto emocional es el elemento central que determinaría la posición ante el objeto de actitud (Williams, Rhodes, y Conner, 2018). Este modelo ha recibido, sin embargo, algunas críticas por parte de autores que consideran necesario diferenciar entre actitud cognitiva y actitud emocional (Elliott, et al, 2013; Lawton, Conner, y McEachan, 2009), y que estarían más de acuerdo con un modelo bidimensional que incluyera un componente cognitivo y un componente afectivo (Wilson, Lindsey y Schooler, 2000).

Ahora bien, independientemente del modelo teórico asumido, hay un consenso en considerar que la base de la discriminación se encuentra en los estereotipos o creencias y en los prejuicios o emociones que suscitan las personas pertenecientes al grupo discriminado. En un nivel más pragmático, asumimos, por tanto, con Pallí y Martínez (2004, p.195) que 
no resulta tan esencial optar por un modelo concreto, como entender que solo podemos hablar de actitud cuando el objeto sobre el cual opinamos, sentimos, o reaccionamos nos afecta, cuando hay un compromiso de implicación personal; es decir, hablamos de actitud cuando nos posicionamos a favor o en contra de un objeto con sentimientos positivos o negativos.

La valoración negativa o prejuicio sobre un grupo, con base en el estereotipo que tenemos sobre este, nos lleva a actuar de un modo determinado respecto a ese grupo o persona, lo que da como resultado la discriminación. Tal y como lo señala Del Águila (2013): La discriminación por razones de discapacidad no se expresa de una manera consciente ni mal intencionada, sino que se basa y se alimenta de una serie de prejuicios previos que han sido heredados de tiempos remotos y que se han convertido en un sustrato cultural que los hace inconscientes. Por eso, es importante desarrollar acciones de educación pública que combatan esta forma negativa de ver y valorar a las personas con discapacidad y, que ayuden a comprender la necesidad de luchar contra la discriminación. (p. 77)

En este sentido, la Convención sobre los Derechos de las Personas con Discapacidad (Naciones Unidas, 2006) establece que:

Se entenderá cualquier distinción, exclusión o restricción por motivos de discapacidad que tenga el propósito o el efecto de obstaculizar o dejar sin efecto el reconocimiento, goce o ejercicio, en igualdad de condiciones, de todos los derechos humanos y libertades fundamentales en los ámbitos político, económico, social, cultural, civil o de otro tipo. Incluye todas las formas de discriminación, entre ellas, la denegación de ajustes razonables. (p. 5)

En términos generales, asumimos, como equipo de investigación, que el prejuicio es un producto social y cultural que se traduce en exclusión. En este sentido Pettigrew y Meertens (1997; 2001) al referirse a los prejuicios étnicos y raciales, diferencian los prejuicios en manifiestos y sutiles, y señalan que ambos están estrechamente relacionados. Estos autores afirman que el prejuicio sutil va más allá de las normas establecidas que proscriben expresiones manifiestas del prejuicio y de la discriminación, e indican que lo sutil es una forma "socialmente aceptable de expresar un prejuicio sin que este sea percibido como un 
sesgo revelador" (Pettigrew y Merteens, 1997, p. 3). Vargas (2013), siguiendo a Pettigrew y Merteens (2001), se centra en los prejuicios sutiles hacia las PcD y concluye que son social y culturalmente construidos y que prevalecen en una población académica en un contexto universitario específico en Costa Rica (Vargas, 2013, p.48). También muestra que es posible identificar los prejuicios sutiles hacia las PcD por medio del análisis crítico del discurso (Iñiguez, 2006; van Dijk, 1984) en sus distintos niveles (retórico- argumentativo, semántico y pragmático-discursivo). El trabajo de Vargas (2013) parte de la definición de discapacidad aportada por la Convención de los Derechos de las Personas con Discapacidad (Naciones Unidas, 2006):

Las personas con discapacidad incluyen aquellas que tengan deficiencias físicas, mentales, intelectuales o sensoriales a largo plazo que, al interactuar con diversas barreras, puedan impedir su participación plena y efectiva en la sociedad, en igualdad de condiciones con las demás. (p.4)

En síntesis, en el trabajo mencionado se concluye que los prejuicios sutiles hacia las personas PcD: a) no se expresan explícitamente, b) la persona que los tiene no los reconoce abiertamente, c) se contradicen en el discurso y la práctica, d) se relacionan con un sentimiento, juicio o preconcepción, e) los prejuicios tienen una carga afectiva, generalmente, con connotación negativa, por lo que pueden provocar emociones como rechazo, desprecio o temor, y f) marcan una diferencia entre la persona que los tiene y la otra persona a quien se le atribuye el juicio o sentimiento.

En este trabajo, la conceptualización del prejuicio sutil hacia la discapacidad fue enriquecida con los aportes de Glick y Fiske (1996; 2001) quienes consideran el prejuicio sexual o sexismo como un fenómeno ambivalente que incluye dos dimensiones: hostil y benevolente (Fiske, 2018). La dimensión hostil, en el caso de la discapacidad, se referiría a los aspectos relacionados con la visión del déficit, la carencia y las limitaciones con que es percibida. La dimensión benevolente, en cambio, estaría relacionada con una visión de debilidad, necesidad de protección y cuido especial requerido por estas personas. Al categorizar una condición particular de diversidad funcional de la persona, se generaliza la discapacidad a toda la persona. La dimensión benevolente es socialmente más aceptada o "políticamente correcta", ya que aparentemente expresa una actitud positiva, pero en ella subyace la misma consideración estereotipada que en la dimensión hostil. La persona queda subordinada a la condición de discapacidad como característica identitaria, es decir, 
prevalece la condición de discapacidad sobre la condición de persona, como aspecto sobresaliente (Wright, 1983) y marca la diferencia entre el endogrupo y el exogrupo lo que genera el prejuicio.

La conceptualización ambivalente del prejuicio hacia las personas con discapacidad ha recibido el nombre de capacitismo (Nario-Redmon, 2010) y ha sido investigada desde la experiencia de las personas con discapacidad (Nario-Redmon, Kemerling, y Silverman, 2019). Las entrevistas reportadas en este trabajo, con una muestra internacional de 185 participantes, confirman que las personas con discapacidad experimentan el prejuicio o capacitismo en múltiples formas: desde las aparentemente más benévolas hasta formas abiertamente hostiles y ambivalentes, o mixtas. Así, por ejemplo, se relatan experiencias de comportamientos paternalistas y condescendientes que menoscaban la autoestima de las personas con discapacidad, y formas hostiles como culpabilización por beneficiarse de políticas que les reportarían privilegios injustificados. Los efectos del capacitismo afectan tanto la percepción de las cualidades afectivas como la percepción de las capacidades de competencia de las personas con discapacidad (Rohmer y Louvet, 2018).

Ahora bien, las categorizaciones por las cuales se produce el prejuicio no actúan aisladamente, sino que se entrecruzan unas con otra. El sexo actúa también como marcador de prejuicio, como han mostrado los estudios de género de las últimas décadas. Por tanto, es acertado analizar si el prejuicio hacia las PcD interactúa con el prejuicio sexual en la línea en la que Soler, Teixeira y Jaime (2008) sostienen. Esto es, que "ser mujer con discapacidad marca una trayectoria de doble discriminación, como mujer y como discapacitada, y añade barreras que dificultan el ejercicio de derechos y responsabilidades como personas" (p. 2). Este aspecto de doble discriminación señalado por la teoría es tomado en este trabajo como otra dimensión a evaluar en una escala de prejuicio hacia las personas con discapacidad. A partir de escalas existentes de sexismo se reformulan ítemes adaptándolos a la condición de discapacidad, dado que el género como categoría analítica permitiría constatar que las mujeres con discapacidad sufren una doble discriminación que las situaría en una posición desigual no solo con respecto a la población en general, sino también con respecto a hombres con discapacidad en particular. Como acota Delgado (2019):

...a fin de cuentas, no lo olvidemos, es lo que da legitimidad al proceso utilizado para generar conocimiento, resulta insostenible la exclusión de categorías analíticas como el género, cuyo potencial interdisciplinarmente explicativo es imprescindible para la comprensión del comportamiento humano. (p. 2) 
La doble discriminación de las mujeres con alguna condición de discapacidad ha sido objeto de investigaciones que confirman el efecto amplificador del sexismo sobre el prejuicio hacia la discapacidad (Froschi, Rubin, y Sprung, 1999; Kabia, et al, 2018; McCall, 2005; McDonald et al., 2007). Como resultado de estos estudios, el Parlamento Europeo recomendó, en su informe de 2013, que las políticas sobre discapacidad "necesitan acciones combinadas que concienticen a la población de la necesidad del cambio de estereotipos, para remover las barreras estructurales y culturales, trabajando la discapacidad conjuntamente con los movimientos feministas" (Davaki, et al, 2013, p.71).

En la siguiente sección se expone la ruta seguida para construir el instrumento a partir de los supuestos teóricos expuestos.

\section{Metodología}

\subsection{Enfoque}

Se siguió el itinerario metodológico habitual para el diseño de escalas psicométricas centradas en los sujetos (Delgado, 2014) se adoptó la integración de resultados cualitativos de estudios previos, con metodología cuantitativa específica para el diseño de instrumentos de evaluación (Cresweel y Plano Clark, 2011). Esta metodología, adoptada en trabajos previos (Delgado y Sánchez-Prada, 2018) permite transformar enunciados discursivos producidos espontáneamente en contextos naturales mediante técnicas cualitativas, en ítems de escalas psicométricas para su utilización en muestras amplias.

En este estudio, para la elaboración del instrumento, se partió del resultado de la investigación cualitativa (Vargas, 2013) y del modelo de prejuicio ambivalente para la evaluación del sexismo (Glick y Fiske, 1996; 2001). A partir de estos supuestos metodológicos, se utilizó el diseño instrumental (Montero y León, 2007; Ato, López, y Benavente, 2013) que tiene como objetivo específico la elaboración de instrumentos de evaluación.

\subsection{Unidades de análisis}

Se invitó a participar en el estudio, voluntaria y anónimamente, a personal de la Universidad de Costa Rica (UCR), Universidad Nacional de Costa Rica (UNA) y Universidad Pontificia de Salamanca (UPSA). Esta selección no aleatoria por muestreo incidental, es adecuado para estudios instrumentales, que tienen como objeto analizar la calidad psicométrica de un instrumento (Montero y León, 2007), y se debe realizar para seleccionar 
muestras de caractrerísticas similares a la población objeto de estudio (AERA, NCME, y APA, 2014; 2018). La muestra reclutada por muestreo incidental no probabilístico quedó integrada por 551 personas, lo cual se ajusta a la recomendación de disponer de 7-8 sujetos por ítem, para análisis psicométricos. La aplicación de la versión inicial del instrumento se efectuó en distintos periodos lectivos durante los años 2018-2019 con el debido consentimiento informado. Las características sociodemográficas de la muestra se exponen en la Tabla 1:

Tabla 1. Características de participantes en la muestra piloto $(n=551)$

\begin{tabular}{llrr}
\hline & Características & \multicolumn{3}{c}{ País } \\
& Mínimo & & España \\
$(\mathrm{N}=342)$ & & $(\mathrm{N}=209)$ & \\
\hline \multirow{3}{*}{ Edad } & Máximo & 71 & 18 \\
& Media & 36.13 & 51 \\
& Desviación Típica & 13.62 & 19.82 \\
& Hombres & $104(30.4 \%)$ & $40(19.1 \%)$ \\
\multirow{2}{*}{ Sexo } & Mujeres & $238(69.6 \%)$ & $169(80.9 \%)$ \\
\hline \multirow{2}{*}{ Ideas } & Derecha & $42(12.3 \%)$ & $33(15.8 \%)$ \\
políticas & Centro & $224(65.5 \%)$ & $99(47.4 \%)$ \\
& Izquierda & $76(22.2 \%)$ & $77(36.8 \%)$ \\
\hline
\end{tabular}

Fuente: Elaboración propia, 2019

\subsection{Técnicas de recolección}

Para la elaboración del instrumento de evaluación del prejuicio hacia las personas con condición de discapacidad, se siguió el proceso siguiente (Delgado, 2014):

3.3.1 Especificación de indicadores: Puesto que el prejuicio es un constructo no observable directamente, se tomaron los siguientes indicadores, desprendidos de los supuestos teóricos anteriormente señalados: 1) dimensión hostil, 2) dimensión benevolente y 3) dimensión sexista.

La especificación de indicadores permitió redactar ítems para cubrir el dominio del constructo, y se tomaron en cuenta las dos características que deben cumplir los indicadores: a) exhaustividad: cubrir los aspectos relevantes del constructo sin omitir ningún aspecto sustantivo; y b) pertinencia: adecuación para el objetivo del instrumento. 
3.3.2 Redacción de los ítems iniciales: A partir del análisis cualitativo del discurso sobre las PcD (Gurdián y Vargas, 2017) y de otros aportes, tales como escalas previas de sexismo (Glick y Fiske, 1996), se redactó una amplia muestra de ítems para cada uno de los indicadores teniendo en cuenta las recomendaciones habituales para este tipo de escalas (Delgado, 2014): que puedan ser rechazados o aceptados sin ambigüedad, evitar tecnicismos y dobles negaciones, reducir en lo posible el efecto de la deseabilidad social con argumentos aparentemente aceptables en las formulaciones socialmente no-deseables, y balancear a lo largo de la escala ítems formulados de forma favorable y de forma desfavorable para minimizar la tendencia a la aquiescencia.

El listado inicial de 72 ítems fue evaluado por doce especialistas en Antropología, Filosofía, Orientación y Educación Especial, Piscología, Psicometría, Trabajo Social, Evaluación y Medición, Administración de Empresas y Educación. Las personas expertas valoraron aspectos de forma y fondo en cuanto a la adecuación y la relevancia de los ítems para medir el constructo. Tras esta revisión, se eliminaron algunos ítems ambiguos, no comprensibles o con lenguaje ambivalente, y se reformularon otros en el sentido sugerido por los y las expertas hasta alcanzar el acuerdo sobre su adecuación. Esta valoración se tomó como evidencia de validez de contenido de la escala.

\subsubsection{Determinación del formato de respuesta y rango escalar: Siguiendo el} procedimiento de la técnica Likert, se adoptó un rango impar de respuesta para cada ítem. Se eligió este formato para mantener un punto central en el cual situarse, sin forzar a los sujetos a posicionarse en un sentido $u$ otro. Si bien algunos investigadores prefieren un número par de alternativas, precisamente para forzar la posición del sujeto, las investigaciones muestran que las propiedades psicométricas de los items no se alteran, con o sin categoría central, cuando el número de categorías es mayor a tres. Se adoptó, por tanto, un número impar de opciones de respuesta en el formato originalmente propuesto por Likert, por considerar que tiene, además, la ventaja de ser más respetuoso con las personas que responden. En cuanto al número de opciones de respuesta, se eligieron 7 alternativas por tratarse de poblaciones con alto nivel educativo, tal como recomienda Likert (desde $1=$ total desacuerdo, hasta $7=$ total acuerdo). En estos casos, elegir 7 puntos de respuesta en lugar de 5 tiene la ventaja de aumentar la variabilidad de las puntuaciones, mejorando la sensibilidad y las propiedades psicométricas de las escalas (Bisquerra y Pérez-Escoda, 2015). 


\subsection{Procesamiento de análisis}

A partir de los datos de la muestra piloto, se realizó el Análisis de Items para seleccionar aquellos que maximizaran la calidad psicométrica de la escala, empleando el software SPSS y AMOS versión 23.

3.4.1. Índice de discriminación de los ítemes: un ítem tiene poder discriminativo si distingue entre los sujetos que puntúan alto y los que puntúan bajo en la escala. En este caso, si permite distinguir entre sujetos con prejuicio y sujetos sin prejuicio. Entre los diferentes índices disponibles, se utilizó el Índice de Homogeneidad corregida (IHc), que estima la correlación entre el ítem y la puntuación total de la escala, eliminando del cómputo total la puntuación del ítem en cuestión. Se tomó como criterio eliminar los ítems cuyo IHc no alcanzara el valor .30, según las recomendaciones al uso.

3.4.2. Fiabilidad estimada por consistencia interna: Una vez eliminados los items no suficientemente discriminativos se utilizó el método alfa de Cronbach para estimar la fiabilidad de la escala, eliminando iterativamente los items que hacían descender la consistencia interna hasta alcanzar un valor de .85, tal como recomienda Cronbach.

3.4.3. Evidencias de validez: En esta fase de la investigación se obtuvieron dos tipos de evidencias de validez de constructo recomendados por los Standards for Educational and Psychological Testing (AERA, NCME y APA, 2014; 2018):

a) Basadas en el contenido: mediante jueces expertos que evaluaron la pertinencia y adecuación de los items para medir el constructo "prejuicio hacia las personas con discapacidad", tal como se indicó anteriormente.

b) Basadas en la estructura interna de la escala: para comprobar si la estructura factorial de los datos empíricos obtenidos en la muestra piloto se ajustaba a los indicadores teóricamente definidos para la escala. Se realizó, en primer lugar, un Análisis Factorial Exploratorio (AFE) para obtener un primer barrido de factores relevantes en la estructura, cuyo ajuste fue probado mediante Análisis Factorial Confirmatorio (AFC).

\section{Resultados}

Tras la revisión y depuración de los items iniciales de la escala, el Análisis Factorial Exploratorio, realizado por el método de Ejes Principales, arrojó una solución de cuatro 
factores, cada uno de ellos compuesto por seis items. Los datos resultaron adecuados para el análisis, con $\mathrm{KMO}=.911$ y un valor $\mathrm{p}<.001$ en la prueba de esfericidad de Bartlett. Los cuatro factores extraídos explicaron un $44,78 \%$ de la varianza, y los índices de homogeneidad corregida de los 24 ítems respecto a la escala total $(\alpha=.888)$ oscilaron entre .327 y .574. En la Tabla 2 se muestra la solución factorial obtenida y la varianza explicada por cada factor tras aplicar rotación Varimax, así como la consistencia interna de cada uno y los índices de homogeneidad corregida $(\mathrm{IHc})$ de sus respectivos items.

Tabla 2. Solución factorial de la escala de prejuicio hacia PcD

\begin{tabular}{|c|c|c|c|c|c|}
\hline & Factor 1 & Factor 2 & Factor 3 & Factor 4 & $\mathrm{IHC}$ \\
\hline ITEM48 & ,742 & & & & .692 \\
\hline ITEM46 & 734 & & & & .668 \\
\hline ITEM33 & ,685 & & & & .645 \\
\hline ITEM36 & ,630 & & & & .616 \\
\hline ITEM12 & 609 & & & & .571 \\
\hline ITEM38 & ,517 & & & & .509 \\
\hline ITEM31 & & ,756 & & & .675 \\
\hline ITEM43 & & ,723 & & & .687 \\
\hline ITEM11 & & ,655 & & & .635 \\
\hline ITEM25 & & 608 & & & .581 \\
\hline ITEM39 & &, 585 & & & .520 \\
\hline ITEM49 & &, 527 & & & .560 \\
\hline ITEM15 & & & 678 & & .634 \\
\hline ITEM27 & & & ,629 & & .588 \\
\hline ITEM24 & & &, 593 & & .576 \\
\hline ITEM14 & & &, 582 & & .547 \\
\hline ITEM20 & & &, 575 & & .576 \\
\hline ITEM06 & & & ,510 & & .505 \\
\hline ITEM21 & & & & ,619 & .553 \\
\hline ITEM23 & & & &, 561 & .578 \\
\hline ITEM07 & & & & ,535 & .541 \\
\hline ITEM04 & & & &, 514 & .495 \\
\hline ITEM18 & & & & ,469 & .518 \\
\hline ITEM13 & & & & ,466 & .465 \\
\hline $\begin{array}{l}\text { Varianza } \\
\text { explicada }\end{array}$ & $12,27 \%$ & $12,05 \%$ & $11,11 \%$ & $9,36 \%$ & \\
\hline $\begin{array}{l}\text { Alfa de } \\
\text { Cronbach }\end{array}$ & .837 & .828 & .809 & .777 & \\
\hline
\end{tabular}

Fuente: Elaboración propia, 2019

Se realizó un Análisis Factorial Confirmatorio por el método Unweighted Least Squares (ULS) (Mc Donald, 1982). Se tomó este método de estimación por ser el recomendado para variables categóricas y ordinales, como es nuestro caso, ya que con este tipo de variables 
se proporciona estimaciones más precisas de los parámetros, al no requerir que los datos observados sigan una distribución determinada.

Para valorar el ajuste resultante del análisis, se utilizaron múltiples criterios recomendados en la literatura sobre el tema (Anderson y Gerbig, 1984; Hu y Bentler, 1999; Schermelleh-Engel, Moosbrugger, y Müller, 2003). Como índices de ajuste, se tomaron: GFI $>$.95; AGFI > .90; NFI > .95; y como índice de error, SRMR < .05. Para la evaluación de la parsimonia, los índices PGFI y PNFI, se consideran aceptables a partir de valores de .50, cuando otros índices de bondad de ajuste son mayores o iguales a .90 (Mulaik et al., 1989). En la Tabla 3 se muestran los resultados obtenidos con un excelente ajuste de los datos al modelo:

Tabla 3. Índices de ajuste para el modelo de prejuicio hacia PcD

\begin{tabular}{lcr}
\hline Índices Ajuste & Valores de ajuste & Modelo de Prejuicio \\
\hline GFI & $>.95$ & .983 \\
AGFI & $>.90$ & .979 \\
NFI & $>.95$ & .972 \\
SRMR & $<.05$ & .045 \\
PGFI & $>.50$ & .806 \\
PNFI & $>.50$ & .866 \\
\hline \multicolumn{2}{c}{}
\end{tabular}

La estructura del modelo contrastado se muestra en la Figura 1: 
Figura 1

Path Diagram del Modelo de Prejuicio hacia las PcD

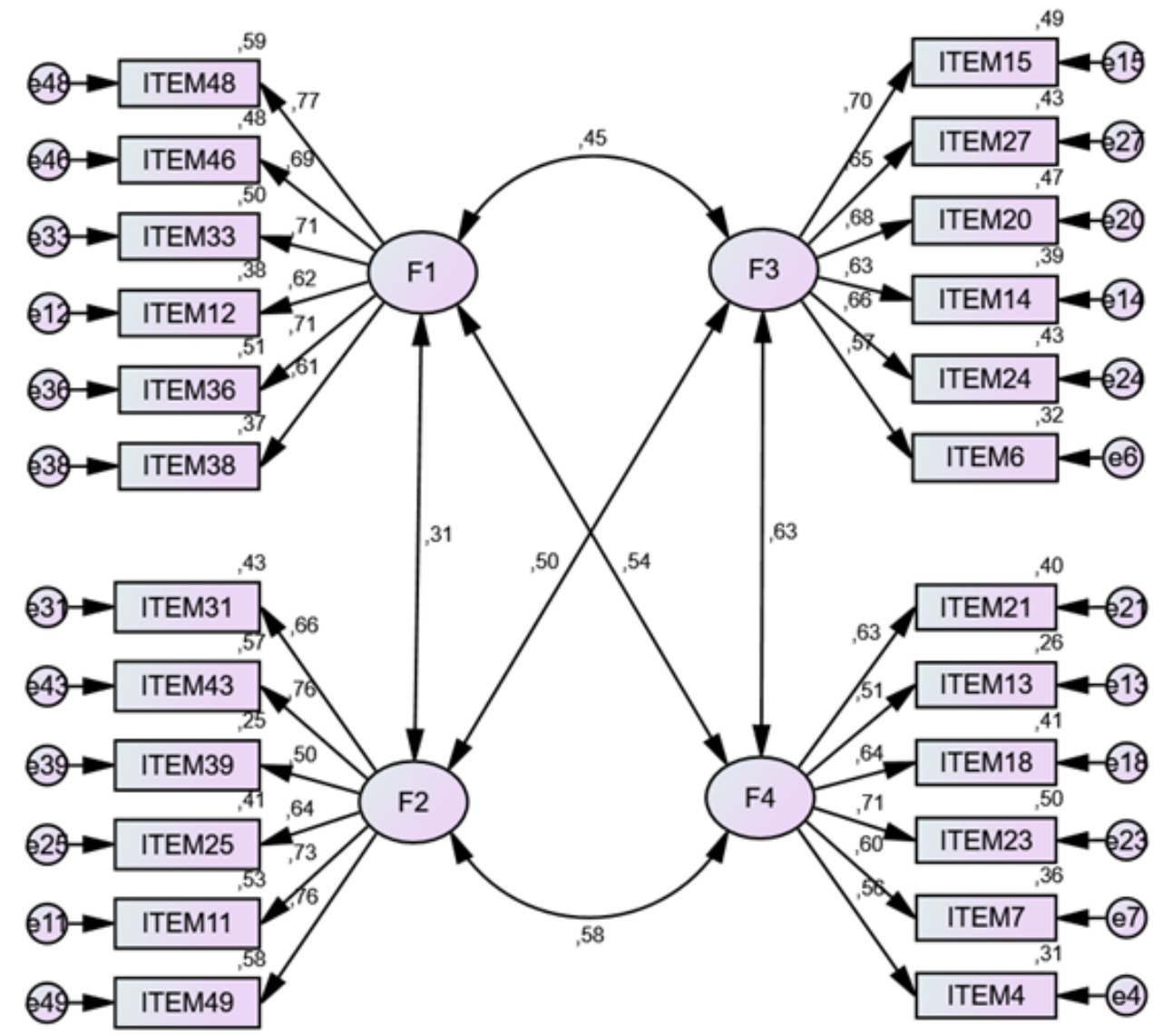

Fuente: Elaboración propia,2019

Como se observa en la Figura 1, este modelo de prejuicio hacia las PcD está compuesto por 4 factores que deberían medir los 3 indicadores definidos teóricamente para la escala. Cada factor está representado por 6 items, con una consistencia interna satisfactoria en todos los factores (véase la Tabla 2).

Los resultados obtenidos permiten, por tanto, confirmar un adecuado ajuste del modelo a los datos, lo que constituye una evidencia de validez del instrumento basada en la estructura interna de la escala, tal como señalan los Standards for Educational and Psychological Testing (AERA, NCME, y APA, 2014; 2018). A partir del pool de 72 items redactados inicialmente, tras el proceso de depuración del contenido y del análisis de items sobre los datos de la muestra piloto, se llegó a una escala de 24 items distribuidos en cuatro factores o dimensiones con cargas satisfactorias en su respectivo factor: entre .50 para el ítem 39 del factor 2, y .77 para el ítem 48 del factor 1 (véase la Figura 1). Esta carga factorial 
satisfactoria confirma la pertinencia de los items para representar el factor, y la fiabilidad estimada por alfa de Cronbach resulta igualmente satisfactoria para los cuatro factores. Si bien, no alcanza el valor .85 recomendado por Cronbach, supera el valor .70 por lo que puede considerarse satisfactorio teniendo en cuenta, además, que cada factor está representado por solo seis items (Hair et al., 2001).

Tabla 4. Factores empíricos del modelo teórico de prejuicio hacia las PcD

\begin{tabular}{lll}
\hline Dimensión & Factores empíricos & Alfa Cronbach \\
\hline Hostil & Factor 2: Evitación de Contacto & .828 \\
& Ej: En general, me incomoda interactuar con una PcD & \\
\cline { 2 - 3 } & Factor 4: Exceso de Demandas & .777 \\
& $\begin{array}{l}\text { Ej: A las PcD hay que ayudarlas demasiado para que salgan } \\
\text { adelante }\end{array}$ & \\
\hline Benevolente & $\begin{array}{l}\text { Factor 1: Idealización Benevolente } \\
\text { Ej: Las PcD tienden a ser más compasivas por su } \\
\text { discapacidad. }\end{array}$ & \\
\hline Sexista & $\begin{array}{l}\text { Factor 3: Amplificación Sexista } \\
\text { Ej: Probablemente las mujeres sufren la discapacidad más }\end{array}$ & \\
& $\begin{array}{l}\text { que los hombres, pues para ellas es más importante el } \\
\text { atractivo físico }\end{array}$ & \\
&
\end{tabular}

Fuente: Elaboración propia, 2019

Tal como se muestra en la Tabla 4, los cuatro factores obtenidos empíricamente se corresponden con las tres dimensiones teóricas del modelo propuesto, e introduce un matiz en la dimensión hostil al identificar dos aspectos diferentes de la hostilidad: "evitación de contacto" (factor 2), de carácter más evaluativo-emocional, y "exceso de demandas" (factor 4), de carácter más evaluativo-cognitivo en la línea propuesta por Lawton y McEachan (2009). La dimensión de "idealización benevolente" (factor 1) confirma la dimensión benevolente o admirativa, identificada como una forma de capacitismo aparentemente positiva, pero igualmente deshumanizante (Nario-Redmon, Kemerling, y Silverman, 2019). Por último, la "amplificación sexista" del prejuicio añade un aspecto no considerado en las escalas al uso, que confirma la interrelación del género con el capacitismo o prejuicio hacia la discapacidad (de Silva, 2008; Froschi, Rubin y Sprung, 1999; Kabia et al., 2018; Rhomer y Louvet, 2018).

Además, la interrelación entre factores es moderada, lo que confirma que las diferentes dimensiones miden aspectos relacionados del prejuicio, pero diferenciados entre sí, según la hipótesis teórica. Tal como se observa en la Figura 1, la correlación más baja (.31) se obtiene entre la dimensión benevolente (factor 1: "idealización benevolente") y la dimensión hostil de carácter más afectivo (factor 2: "evitación de contacto"). La correlación 
más alta (.63) se obtiene entre la dimensión sexista (factor 3: "amplificación sexista") y la dimensión hostil más evaluativa (factor 4: "exceso de demandas").

Otro aspecto relevante para la validez de la escala es el resultado del análisis de invarianza entre países. Este análisis somete a contrastación, si la estructura obtenida se mantiene idéntica para los dos países participantes en la investigación. Los resultados obtenidos confirmaron una estructura factorial idéntica para Costa Rica y España, lo que avala la validez cultural del instrumento (Solano-Flores y Milbourn, 2016). Este resultado abre una importante línea de investigación sobre la estructura profunda del prejuicio en la universidad hacia las PcD. De confirmarse en otros países y regiones diferentes esta equivalencia de la estructura factorial, el instrumento no solo permitiría hacer comparaciones, sino que permitiría, además, realizar contribuciones teóricas sobre una configuración meta-cultural del prejuicio.

Los resultados de los análisis realizados en el proceso de elaboración del instrumento confirman, en primer lugar, que el prejuicio hacia las PcD en el ámbito universitario es multidimensional como ocurre con otro tipo de prejuicios. El ajuste del modelo obtenido a la conceptualización teórica del prejuicio hacia las PcD así lo confirma.

El modelo ambivalente puesto a prueba en esta investigación confirma que la forma hostil del prejuicio, característica de culturas en las que pervive el discurso de la inferioridad, convive con la forma benevolente, más propia de culturas en las que hay una adaptación del discurso a los requerimientos de la igualdad, sin que ello implique una asimilación real de los valores de la igualdad. La "idealización benevolente" de las PcD opera como mecanismo de compensación para hacer más aceptable la "marca de la diferencia" con la que se sigue categorizando, y por tanto, estigmatizando a estas personas (Nario-Redmon, Kemerling, y Silverman, 2019). Este mecanismo de idealización trata de ofrecer al propio sujeto prejuiciado una visión aceptable de sí mismo, transformando en algo aparentemente "sublime" la "marca" a partir de la cual se elabora una representación estigmatizada y colectiva de las personas en razón de su discapacidad. La PcD no es percibida en su singularidad, con la variabilidad de valores y características de las personas que integran cualquier colectivo, sino que se le atribuye, en función de su pertenencia al grupo, una serie de rasgos específicos; en este caso "idealizados" en un discurso de la excelencia que revela la no consideración de igualdad.

También, por un lado, es pertinente destacar que la dimensión hostil del prejuicio presenta una riqueza de contenido que la diversifica en dos factores. Por un lado, emerge 
un factor de carácter más afectivo o menos racionalizado: "evitación de contacto". Y por otro lado, emerge un factor de carácter evaluativo que aglutina una serie de juicios negativos que da soporte racional a la hostilidad hacia las $\mathrm{PcD}$, pero que requiere algún tipo de justificación para no crear conflicto a la persona prejuiciada: "exceso de demandas". En este factor se concentran una serie de juicios irracionales sobre el exceso de atención y recursos desmedidos que demandan las PcD en detrimento de las demás personas. La presencia de dos factores de hostilidad estaría indicando que esta dimensión del prejuicio sigue teniendo mayor representación mental en el ámbito universitario.

Otro aspecto de interés en este modelo de prejuicio hacia las PcD es la confirmación de la interrelacción entre prejuicios, tal como muestra la obtención de un factor específico de "amplificación sexista" del prejuicio hacia las PcD. Este factor específico revela que, en el caso de las mujeres, a la discriminación por razón de su condición de discapacidad se superpone la discriminación por su condición de mujer, como han mostrado otras investigaciones (De Silva, 2008; Froschi, 1999; Kabia et al., 2019; Romer y Louvet, 2009).

Es relevante destacar que la mayor correlación entre factores (.63) se da precisamente entre este factor de "amplificación sexista" (factor 3) y el factor hostil de "exceso de demandas" (factor 4). Si elevamos al cuadrado este valor de correlación directa, obtenemos que ambos factores comparten aproximadamente un $39.69 \%$ de varianza. Esto es, hay una covariación de casi el $40 \%$ entre el juicio hostil sobre la "carga" que suponen las PcD y el sexismo añadido en el caso de las mujeres con discapacidad, a quienes se considera más incapaces y problemáticas que los hombres con esa misma condición.

Las personas que tienden a tener altas puntuaciones en "exceso de demandas", en buena medida tienden a tener puntuaciones también altas en "amplificación sexista" del prejuicio y a la inversa. ¿Cómo podríamos explicar esta interrelación? Una hipótesis plausible es que ambas dimensiones comparten "juicios" de inferioridad por parte de las personas prejuiciadas sobre las personas a las que se aplica el prejuicio (personas con discapacidad y mujeres). Esta interrelación denotaría, por tanto, un marco mental rígido de “jerarquización” de las personas en función de su categorización en grupos estigmatizados o socialmente devaluados que, posiblemente sería común a otras formas de categorización, como marca racial, diversidad sexual, etc.

Esta relación evidencia el interés de investigar discapacidad y género conjuntamente, ya que permitirá conocer la realidad del colectivo de mujeres estudiantes universitarias que llevan además la etiqueta de discapacitadas y que son invisibilizadas en las escalas de 
prejuicio hacia las PcD. La mujer estudiante con discapacidad ha permanecido y permanece, en gran medida, "invisible" no solo para la sociedad, sino también para las instituciones universitarias. Como consecuencia se abre un gran reto para cualquier proyecto universitario que aspire a ser realmente inclusivo, más allá de una declaración de buenas intenciones.

\section{Conclusiones}

Los prejuicios afectan a las PcD en el contexto de las instituciones educativas, aun cuando es posible que se hayan tornado más sutiles y silenciosos, y permanezcan encubiertos dentro del discurso de la igualdad de oportunidades en el marco de la educación inclusiva. Las personas con discapacidad son sistemáticamente asociadas a menor competencia (Rhomer y Louvet, 2016), y suscitan reacciones hostiles cuando se perciben las políticas inclusivas como discriminación positiva injustificada (Nario-Redmon y Oleson, 2016). Así, por ejemplo, quienes ven a las PCD como merecedoras de lástima, generalmente albergan sentimientos de superioridad que contribuyen al abandono, la exclusión y la explotación. En estos casos, se atribuyen los éxitos de estas personas a "privilegios especiales" y "ventajas injustas", lo que genera resentimiento y rechazo (Sherry, 2016). El capacitismo o prejuicio hacia la discapacidad adquiere formas hostiles 0 adversas, paternalistas o condescendientes, benevolentes o admirativas, y envidiosas o celosas, pero todas ellas deshumanizantes (Nario-Redmon, Kemerling, y Silverman, 2019).

Los sectores académicos no son ajenos a estas formas de prejuicio. De ahí la importancia de contar con un instrumento que permita identificar las formas específicas que adquieren los prejuicios en el contexto de la educación superior, y cuantificar su incidencia en los distintos sectores. Los análisis realizados con las muestras estudiadas confirman que el instrumento diseñado reproduce una estructura multidimensional de prejuicio, coherente con el modelo ambivalente de capacitismo experimentado por las personas con discapacidad (Nario-Redmon, Kemerling, y Silverman, 2019).

Esta estructura multidimensional incluye dos componentes hostiles: uno evaluativocognitivo, de "exceso de demandas" y otro evaluativo-afectivo, de "evitación de contacto". Esto, en línea con el modelo bidimensional de actitud (Elliott, et al., 2013). El componente benevolente o admirativo de "idealización benevolente" está en consonancia con el modelo ambivalente de actitud (Fiske, 2018), y el componente de "amplificación sexista" del prejuicio, confirma la interacción entre el prejuicio capacitista y el prejuicio de género 
encontrado en otras investigaciones (Coleman, Brunell, y Haugen, 2015). Se trata, por tanto, de un instrumento que reproduce las dimensiones de las conceptualizaciones más actuales del capacitismo, lo que constituye una evidencia de su validez de constructo basada en la estructura interna. Esta estructura, asegura, además, la validez cultural del instrumento (Solano-Flores y Milbourn, 2016), al haberse confirmado la invarianza del modelo que reproduce una estructura idéntica para medios culturales diferentes como Costa Rica y España. Este resultado abre una importante línea de investigación, ya que de confirmarse esta equivalencia de la estructura factorial en otros medios culturales, el instrumento no solo permitiría hacer comparaciones, sino que permitiría, además, realizar contribuciones teóricas sobre una configuración meta-cultural del prejuicio.

La emergencia del factor de "amplificación sexista" constituye un aporte novedoso en la evaluación del capacitismo al incorporar a la medición, la interacción con el género, tal como recomiendan los informes del Parlamento Europeo (Davaki, et al., 2013). Es interesante notar que la mayor correlación entre factores (.63) se da precisamente entre este factor de "amplificación sexista" (factor 3) y el factor hostil cognitivo de "exceso de demandas" (factor 4). Si elevamos al cuadrado este valor de correlación directa obtenemos que ambos factores comparten aproximadamente un $39.69 \%$ de varianza, lo cual quiere decir que hay una covariación de casi el $40 \%$ entre el juicio hostil sobre la "carga" que suponen las PcD y el sexismo añadido en el caso de las mujeres con discapacidad, a quienes se considera más incapaces y problemáticas que los hombres con esa misma condición. Una hipótesis plausible de esta relación es que ambas dimensiones comparten juicios de "inferioridad" por parte de las personas prejuiciadas sobre las personas a las que se aplica el prejuicio.

El instrumento obtenido supone, una contribución a la investigación y a la evaluación de prejuicios silenciosos, muchas veces no conscientes para las propias personas que los asimilan y reproducen. A la vez, es un medio, que puede contribuir a develar y abordar los prejuicios en sesiones de sensibilización y capacitación, cuyo propósito fuera promover cambios en las creencias y actitudes para contrarrestar la discriminación y exclusión que, en este caso, sufren las personas con alguna condición de discapacidad. También es un recurso disponible para profundizar en el estudio de la presencia de este prejuicio en la universidad, así como un instrumento para avanzar en la investigación teórica sobre este tema. 
Si bien se pueden reseñar algunas limitaciones relacionadas con el tipo de muestreo y número de participantes, así como la necesidad de acumular nuevas evidencias de validez, tales como las basadas en la relación con otras variables (utilidad diagnóstica y pronóstica), estos resultados constituyen un punto de partida alentador para realizar futuros estudios que permitan obtener nuevas evidencias de validez y probar su utilidad para la evaluación.

Se abren, así, múltiples opciones para futuros estudios, entre ellas: realizar diagnósticos institucionales, validación de la escala en otros países y en otros idiomas, elaboración de escalas específicas para atender demandas y necesidades específicas en la erradicación del prejuicio hacia tipos específicos de discapacidad y, evidentemente, estudios de adaptación transcultural, validación y valoración de las propiedades psicométricas de esta versión de la escala ${ }^{6}$.

Por último, señalar que este estudio no está exento de limitaciones, como toda investigación. En primer lugar, la muestra no es representativa. Si bien, en estudios metodológicos como este no es necesaria una representación aleatoria de la población, como ya se señaló en el apartado metodológico (Ato, López, y Bevanente, 2013; Montero y León, 2007), para una evaluación del alcance del prejuicio es deseable aproximarse lo más posible a un muestreo representativo de la población. En segundo lugar, aunque el porcentaje de respuesta a las encuestas se suele estimar en torno al $20 \%$, la respuesta de la población no tuvo el nivel de participación deseado.

Asimismo, a la dificultad de obtener participación en la población, se suma la dificultad de la resistencia a responder cuestionarios cuando el objeto de investigación está impregnado de deseabilidad social, como es el caso de cualquier tipo de prejuicios. Estudios con muestras de mayor tamaño y mayor representatividad de la población universitaria permitirán someter a prueba la generalización de estos resultados que, en principio, resultan promisorios tanto por la adecuación al modelo teórico de capacitismo como por la adecuación de los índices de ajuste obtenidos.

Imposible finalizar sin agradecer, en primer lugar, a todas y cada una de las personas que voluntaria y desinteresadamente respondieron el cuestionario inicial de más de 70 ítems, y sin cuyas respuestas no habría sido posible hacer esta investigación. En segundo lugar, agradecemos a las y los jueces que participaron en la fase de validación del contenido. A ellos y a ellas, hacerles constar que sus aportes en dicha fase fueron muy

${ }^{6}$ Se puede solicitar la escala para investigaciones o estudios específicos a la siguiente dirección electrónica: alicia.gurdian@ucr.ac.cr 
valiosos. En tercer lugar, agradecemos al personal del Instituto de Investigación en Educación (INIE), que estuvo vinculado con el proyecto, muy especialmente al M.Sc. Franklin Rodríguez Vargas, por todo el apoyo informático que nos brindó. Sin el apoyo desinteresado de todas estas personas, no hubiese sido posible terminar con éxito este proyecto.

\section{Referencias}

AERA, NCME and APA. (2014). Standards for Educational and Psychological Testing. Washington, D.C.: American Educational Research Association.

AERA, NCME and APA. (2018). Estándares para pruebas educativas y psicológicas. Washington, D.C.: American Educational Research Association.

Albarracín, Dolores and Shavitt, Sharon. (2018). Attitudes and attitude change. Annual Review of Psychology, 69, 299-327. doi: https://doi.org/10.1146/annurev-psych$\underline{122216-011911}$

Allport, Gordon W. (1935). Attitudes. En C. Murchison (Ed.), Handbook of social psychology (pp. 798-844). Worcester, MA: Clark University Press.

Anderson, James and Gerbig, David. (1984). The effect of sampling error on convergence, improper solutions, and goodness-of-fit indices for maximum likelihood confirmatory analysis. Psychometrika, 49, 155-173.

Ato, Manuel; López, Juan J. and Benavente, Ana. (2013). Un sistema de clasificación de los diseños de investigación en psicología. Anales de Psicología, 29(3), 1038-1059.

Bisquerra, Rafael and Pérez-Escoda, Nuria. (2015). ¿Pueden las escalas Likert aumentar en sensibilidad? REIRE, Revista d'Innovació i Recerca en Educació, 8(2), 129-147. doi: $\underline{10.1344 / \text { reire2015.8.2.828 }}$

Briñol, Pablo, Petty, Richard E. and Stavraki, María. (2019). Structure and Function of Attitudes. Nueva York: Oxford University Press.

Coleman, Jill M., Brunell, Amy B., and Haugen, Ingrid M. (2015). Multiple Forms of Prejudice: How Gender and Disability Stereotypes Influence Judgments of Disabled Women and Men. Current Psychology, 34, 177-189.

Creswell, John W., and Plano Clark, Vicki. L. (2011). Designing and conducting mixed methods research (2nd ed.). Thousand Oaks, CA: Sage.

Davaki, Konstantina, Marzo, Claire, Naminio, Elisa, and Arvanitidou, Maria. (2013). Discrimination Generated by the Instersection of Gender and Disability. Bruselas: European Parliament: Policy Department C - Citizens' Rights and Constitutional Affairs. http://www.europarl.europa.eu/studies 
De Silva, Rangita. (2008). Disability Rights, Gender, and Development. A Resource Tool for Action. United Nations: Center for Research on Women.

Del Águila, Luis Miguel. (2013). Estereotipos y prejuicios que afectan a las personas con discapacidad Las consecuencias que esto genera para el desarrollo de políticas públicas inclusivas en cualquier lugar del mundo. En P. Rosales, Discapacidad, justicia y Estado. Discriminación, estereotipos y toma de conciencia (Vol. 2, pp. 67-99). Buenos Aires: INFOJUS.

Delgado, Carmen. (2014). Viajando a Ítaca por mares cuantitativos. Manual de ruta para investigar en grado y postgrado. Salamanca: Amarú.

Delgado, Carmen and Sánchez-Prada, Andrés. (2018). Exploring Gender Stereotypes Using Qualitative-Quantitative Methodological Integration. En P. Costa, L.P. Réis, F. Neri de Souza, and A. Moréira (Eds.) Computer Supported Qualitative Research (vol. 621) Advances in Intelligent Systems and Computing (pp. 152-163). Cham (Switzerland): Springer International Publishing AG.

Delgado, Carmen. (2019). La Ceguera al Género Inducida por la Ceguera a los Estándares de Medición. Comentario a Ferrer-Pérez y Bosch-Fiol, 2019. Anuario de Psicología Jurídica, 30(1). Doi https://doi.org/10.5093/apj2019a8

Elliott, Mark A., Thomson, James A., Robertson, Kirsty, Stephenson, Carry and Wicks, John. (2013). Evidence that changes in social cognitions predict changes in self-reported driver behavior: Causal analyses of two-wave panel data. Accident Analysis and Prevention, 50, 905-916. https://doi.org/10.1016/j.aap.2012.07.017

Fishbein, Martin and Ajzen, Icek. (1975). Belief, Attitude, Intention and Behavior: An Introduction to Theory and Research. Boston: Addison-Wesley.

Fiske, Susan. (2018). Social cognition. Londres: Routledge.

Froschi, Merle, Rubin, Ellen, Sprung, Barbara (1999). Connecting Gender and Disability. Newton, MA: Women's Educational Equity Act (WEEA).

Glick, Peter. and Fiske, Susan. (1996). The Ambivalent Sexism Inventory: Differentiating Hostile and Benevolent Sexism. Journal of Personality and Social Psychology, 70, 491512.

Glick, Peter and Fiske, Susan. (2001). An ambivalent alliance: Hostile and benevolent sexism as complementary justifications for gender inequality. American Psychologist, 56(2), 109-118.

Gurdián, Alicia and Vargas, Marie-Claire. (2017). Informe parcial Proyecto Construyendo una escala de prejuicios hacia las personas con discapacidad. San José/Heredia, Costa Rica: INIE-UCR, CIDE-UNA.

Hair, Joseph H., Anderson, Rolph E., Tatham, Ronald L., and Black, William C. (2001). Estadística Multivariante. Madrid: Prentice Hall. 
$\mathrm{Hu}$, Li-tze and Bentler, Peter. (1999). Cut-off criteria for fit indexes in covariance structure analysis: conventional criteria versus new alternatives. Structural Equation Modeling, 6(1), 1-55.

Iñiguez, Lupicinio. (2006). Análisis del discurso. Manual para las ciencias sociales (2 $2^{\text {th }}$ ed.). Barcelona: Editorial UOC.

Kabia, Evelyn, Mbau, Rahab, Muraya, Kelly E., Morgan, Rosemary, Molyneux, Sassy, and Barasa, Edwine. (2018). How do gender and disability influence the ability of the poor to benefit from pro-poor health financing policies in Kenya? An intersectional analysis. International Journal for Equity in Health, 17(149), 1-12. Doi: https://doi.org/10.1186/s12939-018-0853-6

Lawton, Rebecca, Conner, Mark, and McEachan, Rosemary. (2009). Desire or reason: Predicting health behaviors from affective and cognitive attitudes. Health Psychology, 28(1), 56-65. Doi: https://doi.org/10.1037/a0013424

McCall, Leslie. (2005) The Complexity of Intersectionality, Journal of Women in Culture and Society, 30(3), 1771-1800, http://www.journals.uchicago.edu/doi/pdf/10.1086/426800

McCartan, Rebecca, Elliott, Mark A., Pagani, Stefania, Finnegan, Eimear, and Kelly, Steve W. (2018). Testing the effects of explicit and implicit bidimensional attitudes on objectively measured speeding behaviour. British Journal of Social Psychology, 57, 630-651.

McDonald, Katherine E., Keys, Christopher, and Balcazar, Fabricio (2007). Disability, race/ethnicity and gender: themes of cultural oppression, acts of individual resistance. American Journal of Community Psychology, 39, 145-161. Doi: 10.1007/s10464-0079094-3

Mc Donald, Roderick. (1982). Linear versus nonlinear models in item response theory. Applied Psychological Measurement, 6, 379-396.

Montero, Ignacio and León, Orfelio (2007). A guide for naming research studies in psychology. International Journal of Clinical and Health Psychology, 7(3), 847-862.

Mulaik, Stanley A., James, Larry R., Van Alstine, Judith, Bennett, Nathan., Lind, Sherri. \& Stilwell, C. Dean. (1989). Evaluation of goodness-of-fit indices for structural equation models. Psychological Bulletin, 105, 430-445.

Nario-Redmond, Michelle R. (2010). Cultural stereotypes of disabled and non-disabled men and women: Consensus for global category representations and diagnostic domains. British Journal of Social Psychology, 49, 471-488. DOI: 10.1348/014466609X468411

Nario-Redmond, M. R., and Oleson, Kathryn C. (2016). Disability group identification and disability-rights advocacy: Contingencies among emerging and other adults. Emerging Adulthood, 4, 207-218. Doi: https://doi.org/10.1177/2167696815579830 
Nario-Redmond, Michelle R., Kemerling, Alexia A. and Silverman, Arielle (2019). Hostile, Benevolent, and Ambivalent Ableism: Contemporary Manifestations. Journal of Social Issues, 75(3), 726-756. Doi: https://doi.org/10.1111/josi.12337

Organización de las Naciones Unidas. (2006). Convención sobre los Derechos de las Personas con Discapacidad y Protocolo Facultativo. Recuperado de https://www.un.org/development/desa/disabilities-es/convencion-sobre-los-derechosde-las-personas-con-discapacidad-2.html

Pallí, Cristina and Martínez, Luz María (2004). Naturaleza y organización de las actitudes. En Tomás Ibáñez (coord.), Introducción a la psicología social (pp.183-254). Barcelona: UOC.

Pettigrew, Thomas and Meertens, Roel. (1997). Is subtle prejudice really prejudice? Public Opinion Quaterly, 61, 54-71.

Pettigrew, Thomas and Meertens, Roel. (2001). In defense of the subtle prejudice concept: a retort. European Journal of Social Psychology, 31, 299-309.

Quiles del Castillo, María Nieves. (2019). El estigma social. Convivir con la mirada negativa del otro. Madrid: Alianza Editorial.

Rohmer, Odile and Louvet, Eva. (2018). Implicit stereotyping against people with disability. Group Processes and Intergroup Relations, 21(1), 127-140. Doi: https://doi.org/10.1177/1368430216638536

Rosenberg, Milton and Hovland, Carl. (1960). Cognitive, Affective, and Behavioral Components of Attitudes. New Haven: Yale University Press.

Schermelleh-Engel, Karin, Moosbrugger, Helfried and Müller, Hans. (2003). Evaluating the fit of structural equation models: tests of significance and descriptive goodness-of-fit measures. Methods of Psychological Research Online, 8(2), 23-74.

Sherry, Mark. (2016). Disability hate crimes: Does anyone really hate disabled people? New York: Routledge.

Solano-Flores, Guillermo and Milbourn, Tamara. (2016). Capacidad evaluativa, validez cultural y validez consecuencial. RELIEVE, 22(1), M12.

Soler, A., Teixeira T. C. y Jaime V. (2008). Discapacidad y dependencia: una perspectiva de Género. En XI Jornadas de Economía Crítica, ECOCRI, Bilbao. Recuperado de http://www.ucm.es/info/ec/ecocri/eus/Soler Domingo.pdf

Van Dijk, Teun. (1984). Prejudice in discourse. Amsterdam: John Benjamin Publising Co.

Vargas, Marie-Claire. (2013). Influencia de los prejuicios de un sector de la población Universitaria respecto a la discapacidad en la construcción de una cultura institucional inclusiva. Universidad Nacional. Costa Rica, 2012-2013. (Tesis Doctoral) Universidad de Costa Rica, Sede Rodrigo Facio, San José, Costa Rica. 
Williams, David M., Rhodes, Ryan E., and Conner, Mark T. (2018). Affective determinants of health behavior. Nueva York: Oxford University Press.

Wilson, Timothy, Lindsey, Samuel, and Schooler, Tonya Y. (2000). A model of dual attitudes. Psychological Review, 107(1), 101-126. Doi: https://doi.org/10.1037//0033295X.107.1.101

Wright, Beatrice. (1983). Physical Disability-A Psychosocial Approach. NY: Harper and Row Publishers. 
Revista indizada en

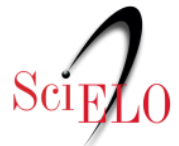

redalyc.sy latindex

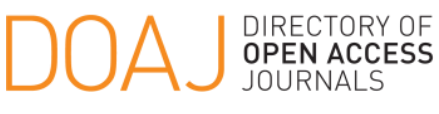

Distribuida en las bases de datos:

- Dialnet

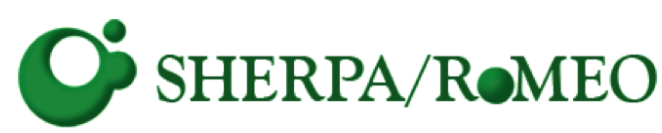

REDIB

Red Iberoamericana

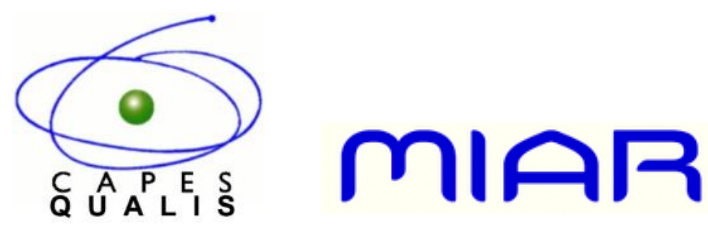

